\title{
MÉTODOS VARIACIONALES EN LA RECUPERACIÓN DE IMÁGENES
}

\section{Víctor Osorio Vidal $^{1}$}

RESUMEN.- Se estudia la minimización de funcionales semicuadráticos de la forma

$$
J_{\alpha}(f)=\int_{\Omega}(p-R f)^{2} d x d y+\alpha \int_{\Omega} \phi(|\nabla q|) d x d y
$$

El funcional en mención aparece en los problemas de recuperación de imágenes cuando una imagen considerada como una función $f: \Omega \subset \mathrm{R}^{2} \rightarrow \mathrm{R}$, que describe una escena real, es observada y reproducida como una función $p: \Omega \subset \mathrm{R}^{2} \rightarrow \mathrm{R}$.

PALABRAS CLAVE.- Recuperación de imágenes, funcionales semicuadráticas, Métodos variacionales convexidad.

\section{VARIATIONAL METHODS IN IMAGE RECOVERY}

ABSTRACT.- We study the semicuadratic minimization problem of the form

$$
J_{\alpha}(f)=\int_{\Omega}(p-R f)^{2} d x d y+\alpha \int_{\Omega} \phi(|\nabla q|) d x d y
$$

this functional arises in image recovery problems, when a image that describe a real scene, consider like a function $f: \Omega \subset \mathrm{R}^{2} \rightarrow \mathrm{R}$, is observed and reproduced like a function $p: \Omega \subset \mathrm{R}^{2} \rightarrow \mathrm{R}$.

KEYWORDS.- Imagen recovery, semicuadratic functionals, variational Methods, convexity.

\section{INTRODUCCIÓN}

En el presente trabajo se estudia la minimización de funcionales semicuadráticos de la forma

$$
J_{\alpha}(f)=\int_{\Omega}(p-R f)^{2} d x d y+\alpha \int_{\Omega} \phi(|\nabla f|) d x d y
$$


variando $f$ en el conjunto $\left\{f \in L^{2}(\Omega) / \nabla f \in L^{1}(\Omega) \times L^{1}(\Omega)\right\}$ que es un subespacio de $L^{2}(\Omega)$. Con este fin se aplican los métodos variacionales, en particular las técnicas de minimización de funcionales convexas, semicontinuas inferiormente y coercivas, luego se hace uso de los resultados clásicos de compacidad (débil, débil*) y de los procesos de diagonalización, con los que se establecen los resultados de existencia, regularidad y acotación para la solución del problema planteado.

El funcional citado aparece en los problemas de recuperación de imágenes cuando una imagen considerada como una función $f: \Omega \subset \mathbb{R}^{2} \rightarrow \mathbb{R}$ que describe una escena real, es observada y reproducida como una función $p: \Omega \subset \mathbb{R}^{2} \rightarrow \mathbb{R}$. En el proceso de captación de la imagen $f$, ésta sufre una degradación y es vista como $p$. El modelo de degradación más común es el lineal de la forma

$$
p-R f=\eta
$$

Donde $\mathrm{R}$ es un operador lineal y $\eta$ representa el error o ruido.

Se establece la existencia de una función $f \in\left\{f \in L^{2}(\Omega) / \nabla f \in L^{1}(\Omega) \times L^{1}(\Omega)\right\}$ que minimiza la funcional $J_{\alpha}(f)$. Imponiendo condiciones adicionales a la función $p$ se deduce la acotación y cierta regularidad para la función minimizante.

\section{PRELIMINARES}

Considerando $f: \Omega \subset \mathbb{R}^{2} \rightarrow \mathbb{R}$ una función que describe una escena real, que es observada y reproducida como una función $p: \Omega \subset \mathbb{R}^{2} \rightarrow \mathbb{R}$. En el proceso de captación de la imagen $f$, ésta puede sufrir una degradación y ser vista como $p$. El modelo de degradación más común es el lineal, es decir, se tiene la siguiente ecuación

$$
p-R f=\eta
$$

donde $R$ es un operador lineal y $\eta$ el error o ruido.

La ecuación (1) plantea dos problemas: primero, el espacio de funciones en que tiene sentido dicha ecuación y en segundo lugar la posibilidad de hallar $f$ de modo que sea lo menor posible. Entonces el modelo propuesto en (1), permite buscar una solución como el mínimo del funcional de energía

$$
J_{\alpha}(f)=\int_{\Omega}(p(x, y)-(R f)(x, y))^{2} d x d y+\alpha \int_{\Omega} \phi(|\nabla f(x, y)|) d x d y .
$$


Donde $R$ es un operador lineal de $L^{2}(\Omega)$ en $L^{2}(\Omega)$, la primera integral representa un término ligado al dato, y $\Phi(f)=\int_{\Omega} \phi(|\nabla f(x, y)|) d x d y$ es el término de regularización, $\phi: \mathbb{R}^{+} \rightarrow \mathbb{R}^{+}$es la función de regularización y $\alpha \in R^{+}$es un parámetro que permite equilibrar la influencia de cada integral en la funcional $J_{\alpha}(f)$. Por ejemplo, si $\alpha=0, J_{\alpha}(f)$ es

$$
J_{0}(f)=\int_{\Omega}(p(x, y)-(R f)(x y))^{2} d x d y
$$

y de esta manera la funcional queda reducida sólo al término ligado a los datos del problema. El problema de minimización del funcional $J_{0}(f)$, esto es; hallar el

$$
\inf _{f \in L^{2}(\Omega)} J_{0}(f)
$$

Las hipótesis que se imponen a $\phi$ son las siguientes:

(H1) La función $\phi: R^{+} \rightarrow R^{+}$es de clase $C^{2}$, no decreciente, y satisface

$$
\phi^{\prime}(0)=0 \quad \text { y } \quad \phi^{\prime \prime}(0)>0 .
$$

(H2) Además tiene la propiedad

$$
\lim _{t \rightarrow+\infty} \phi^{\prime \prime}(t)=\lim _{t \rightarrow+\infty} \frac{\phi^{\prime}(t)}{t}=0 \quad \text { y } \lim _{t \rightarrow+\infty} \frac{\phi^{\prime \prime}(t)}{\frac{\phi^{\prime}(t)}{t}}=0
$$

(H3) Existen constantes $a_{i}>0$ y $b_{i} \geq 0, i=1,2$, tal que

$$
a_{1} t-b_{1} \leq \phi(t) \leq a_{2} t+b_{2} ; \forall t \in R^{+}
$$

(H4) La función $\phi: \mathbb{R}^{+} \rightarrow \mathbb{R}^{+}$es estrictamente convexa.

Será necesario definir la función $\phi$ sobre todo $\mathbb{R}$, para este fin se extiende $\phi$ por paridad a todo $\mathbb{R}$.

Existen muchas funciones $\phi$ que cumplen $(\mathrm{H1})$ - (H4), sin embargo no existe ningún criterio para la elección de $p$. Un ejemplo de esta clase de funciones es $\phi(t)=\sqrt{1+t^{2}}$ la cual verifica $(\mathrm{H} 1)-(\mathrm{H} 4)$. 


\section{REDUCCIÓN DE FUNCIONALES A FUNCIONALES SEMICUADRÁTICAS.}

Veamos como el funcional de regularización

$$
L_{\phi}(f)=\int_{\Omega} \phi(|\nabla f|) d x d y
$$

puede ser representado por el ínfimo de las funciones cuadráticas. Para esto, haremos uso de la transformada de Fenchel - Legendre (o polar). Si $l: \mathbb{R}^{N} \rightarrow \mathbb{R}$, entonces, su transformada de Fenchel - Legendre es la función convexa $l^{*}\left(\xi^{*}\right)$ definida por

$$
l^{*}\left(\xi^{*}\right)=\operatorname{Sup}_{\xi \in \mathbb{R}^{N}}\left\{\left\langle\xi, \xi^{*}\right\rangle-l(\xi)\right\}
$$

donde $\left\langle\xi, \xi^{*}\right\rangle$ es el producto escalar usual. Esta definición puede ser extendida, sin dificultad, a los espacios $L_{N}^{p}=L^{p}(\Omega) \times \ldots \times L^{p}(\Omega) \quad(\mathrm{N}$ veces $)$ y su dual $\left(L_{N}^{p}\right)^{*}=L^{p^{*}}(\Omega) \times \ldots \times L^{p^{*}}(\Omega)$ (N veces) donde $\Omega$ es un conjunto abierto de $\mathbb{R}^{N}$

\section{Funcionales auxiliares $\Phi$ y $\Psi$.}

Usaremos la noción de polaridad en nuestro problema con $N=2, p=p^{*}=2$.

Para $\xi, \xi^{*} \in \mathbb{R}^{2}$, definimos las funciones:

$$
\begin{gathered}
l(\xi)=\frac{|\xi|^{2}}{2}-\phi(|\xi|) \\
\psi\left(\xi^{*}\right)=l^{*}\left(\xi^{*}\right)-\frac{\left|\xi^{*}\right|^{2}}{2} .
\end{gathered}
$$

Así como las funcionales definidas sobre $L_{2}^{2}=L^{2}(\Omega) \times L^{2}(\Omega)$ por

$$
\Phi(u)=\int_{\Omega} \phi(|u(x, y)|) d x d y
$$

la relación (9) se puede escribir como

$$
\Phi(u(x, y))=\int_{\Omega}\left(\frac{\langle u(x, y), u(x, y)\rangle}{2}-l(u(x, y))\right) d x d y
$$




$$
\Psi(v)=\int_{\Omega} \psi(v(x, y)) d x d y
$$

El siguiente teorema cuya demostración se encuentra en Osorio [5] Pág.67 establece que $\Phi$ y $\Psi$ son duales en cierto sentido.

\section{Teorema 3.1.}

La función $\phi$ (extendida por paridad sobre $\mathbb{R}$ ) verifica las hipótesis:

(H3) Existen constantes $a_{i}>0$ y $b_{i} \geq 0, \quad i=1,2$, tal que

$$
a_{1}|t|-b_{1} \leq \phi(t) \leq a_{2}|t|+b_{2} \quad ; \quad \forall t \in \mathbb{R} ;
$$

(H5) La función $t \rightarrow \frac{t^{2}}{2}-\phi(t)$ es convexa sobre $\mathbb{R}$. Entonces

$$
\begin{aligned}
& \Phi(u)=\int_{\Omega} \phi\left(\mid u(x, y \mid) d x d y=\inf _{v \in L_{2}^{2}} \int_{\Omega}\left(\frac{|u-v|^{2}}{2}+\psi(v)\right) d x d y\right. \\
& \Psi(v)=\int_{\Omega} \psi(v(x, y)) d x d y=\operatorname{Sup}_{u \in L_{2}^{2}} \int_{\Omega}\left(-\frac{|u-v|^{2}}{2}+\phi(|u|)\right) d x d y .
\end{aligned}
$$

Se observó de la condición (H3) para $\phi$ que la solución debe buscarse en el espacio

$$
v=\left\{f \in L^{2}(\Omega), \nabla f \in L^{1}(\Omega)^{2}\right\} \subset H^{1}(\Omega)
$$

Para usar la dualidad, buscaremos una solución $f$ en el espacio $H^{1}(\Omega)$.

Usando la relación (11),

$$
J_{\alpha}(f)=\int_{\Omega}(p-R f)^{2} d x d y+\alpha \int_{\Omega} \phi(|\nabla q|) d x d y
$$

se escribe, para , $f \in H^{1}(\Omega)$ como

$$
J_{\alpha}(f)=\int_{\Omega}(p-R f)^{2} d x d y+\alpha \inf _{b \in L_{2}^{2}} \int_{\Omega}\left(\frac{|\nabla f-b|^{2}}{2}+\psi(b)\right) d x d y .
$$

Luego, tomando ínfimos

$$
\inf _{f \in H^{1}(\Omega)} J_{\alpha}(f)=\inf _{f \in H^{1}(\Omega)} \inf _{b \in L_{2}^{2}}\left[\int_{\Omega}(p-R f)^{2} d x d y+\alpha \int_{\Omega}\left(\frac{|\nabla f-b|^{2}}{2}+\psi(b)\right) d x d y\right] .
$$


Reordenando e invirtiendo los ínfimos se obtiene

$$
\inf _{f \in H^{1}(\Omega)} J_{\alpha}(f)=\inf _{b \in L_{2}^{2}}\left[\alpha \int_{\Omega} \psi(b) d x d y+\inf _{f \in H^{1}(\Omega)} \int_{\Omega}\left((p-R f)^{2}+\alpha \frac{|\nabla f-b|^{2}}{2}\right) d x d y\right.
$$

Ahora, fijando $b \in L_{2}^{2}$ se resuelve el problema

$$
\inf _{f \in H^{1}(\Omega)}\left[\int_{\Omega}\left((p-R f)^{2}+\alpha \frac{|\nabla f-b|^{2}}{2}\right) d x d y\right]
$$

\section{TEOREMA DE EXISTENCIA Y UNICIDAD}

Para simplificar, supondremos que $R=I$ sobre $L^{2}(\Omega)$ y $\alpha=1$, lo cual no modifica el estudio teórico del problema. En consecuencia, el funcional que se estudiará es

$$
J(f)=\int_{\Omega}(p-f)^{2} d x d y+\int_{\Omega} \phi(|\nabla f|) d x d y
$$

Asumiendo las hipótesis:

( $\widehat{H} 1$ ) $p \in L^{\infty}(\Omega)$ y $0 \leq p(x, y) \leq 1$ acotado en casi todo punto (a.e) $(x, y) \in \Omega$.

$\left(\widehat{H} 2\right.$ ) $\phi: \mathbb{R} \rightarrow \mathbb{R}$ es par, de clase $C^{2}$, no decreciente sobre $\mathbb{R}^{+}$, y existen constantes $a_{i}>0, b_{i} \geq 0, i=1,2$, tal que

$$
a_{1}|t|-b_{1} \leq \phi(t) \leq a_{2}|t|+b_{2}, \forall t \in \mathbb{R},
$$

( $\widehat{H} 3$ ) $0<\phi^{\prime \prime}(t)<1, \forall t \in \mathbb{R}$.

\section{Proposición 4.1.}

Para b fijo en $L_{2}^{2} y$ para $p$ satisfaciendo $(\widehat{H} 1)$, el problema

$$
J(f)=\inf _{f \in H^{1}(\Omega)} \int_{\Omega}\left((p-f)^{2}+\frac{|b-\nabla f|^{2}}{2}\right) d x d y
$$

tiene una única solución $f_{b} \in H^{1}(\Omega)$; verificando la ecuación de Euler

$$
-\Delta f_{b}+2 f_{b}=2 p-\operatorname{div} b \text { en } D^{\prime}(\Omega)
$$




\section{Prueba.}

Ver Osorio [5], Pág. 73.

De la proposición 4.1, tenemos que; para cada $b \in L_{2}^{2}$ existe un único $f_{b}$ tal que

$$
J_{b}\left(f_{b}\right) \leq J_{b}(f) ; \quad \forall f \in H^{1}(\Omega)
$$

lo cual es equivalente a escribir

$$
\int_{\Omega}\left(f_{b}-p\right)^{2} d x d y+\int_{\Omega} \frac{\left|b-\nabla f_{b}\right|^{2}}{2} d x d y \leq \int_{\Omega}(f-p)^{2} d x d y+\int_{\Omega} \frac{|b-\nabla f|^{2}}{2} d x d y
$$

Adicionando $\psi(b)$ a ambos miembros de la desigualdad (18) y tomando el ínfimo cuando $b$ recorre $L_{2}^{2}$ para todo $f \in H^{1}(\Omega)$, obtenemos,

$$
\begin{gathered}
\inf _{b \in I_{2}^{2}} \int_{\Omega}\left(\left(f_{b}-p\right)^{2}+\int_{\Omega} \frac{\left|b-\nabla f_{b}\right|^{2}}{2}+\psi(b)\right) d x d y \leq \inf _{b \in L_{2}^{2}} \int_{\Omega}\left((f-p)^{2}+\int_{\Omega} \frac{|b-\nabla f|^{2}}{2}+\psi(b)\right) d x d y \\
\leq \int_{\Omega}\left((f-p)^{2}+\phi(|\nabla f|)\right) d x d y=J(f) .
\end{gathered}
$$

Denotando

$$
T(b)=\int_{\Omega}\left(\left(f_{b}-p\right)^{2}+\frac{1}{2}\left|b-\nabla f_{b}\right|^{2}+\psi(b)\right) d x d y=J_{b}\left(f_{b}\right)+\int_{\Omega} \psi(b) d x d y
$$

ahora es necesario probar que el problema $\inf _{b \in L_{2}^{2}} T(b)$ tiene una solución $b_{0}$, la cual involucra la existencia de una función $f_{0}$; solución del problema inicial

$$
J\left(f_{0}\right) \leq J(f), \forall f \in v .
$$

En primer lugar, estableceremos algunas propiedades de la función dual $\psi$.

\section{Lema 4.2.}

Si $\phi$ verifica $(\widehat{H} 2)$ y ( $\widehat{H} 3$ ), entonces la función $\psi$ definida en (8) goza de las siguientes propiedades:

i) $\xi^{*} \rightarrow \psi\left(\xi^{*}\right)$ es estrictamente convexa.

ii) Existen constantes $a_{i}^{\prime}>0$ y $b_{i}^{\prime} \geq 0$; tal que

$$
a_{1}^{\prime}\left|\xi^{*}\right|-b_{1}^{\prime} \leq \psi\left(\xi^{*}\right) \leq a_{2}^{\prime}\left|\xi^{*}\right|+b_{2}^{\prime} ; \forall \xi^{*} \in \mathbb{R}^{2}
$$




\section{Prueba.}

Ver Osorio [5], Pág. 76.

Si $b_{n}$ es una sucesión minimizante, entonces es simple deducir de (18) y (19) que $b_{n}$ y $f_{b_{n}}$ verifican las restricciones

$$
\left\|f_{b_{n}}\right\|_{L_{1}^{2}} \leq c \text { y }\left\|b_{n}\right\|_{L_{2}^{1}} \leq c
$$

donde $c$ es una constante que sólo depende de los datos. Pero no es posible obtener una estimación para $f_{b_{n}}$ en la norma de $H^{1}(\Omega)$ y para $b_{n}$ en $L^{2}(\Omega)^{2}$. Por tanto, debemos trabajar sobre el espacio no reflexivo $L^{1}(\Omega)$ o sobre el espacio de medidas acotadas $M_{b}(\Omega)$.

Para superar esta dificultad, regularizamos el problema haciendo una ligera modificación sobre el potencial $\phi$.

Definimos la función

$$
\phi_{\varepsilon}(t)=\phi(t)+\frac{\varepsilon}{2} t^{2}, \varepsilon>0
$$

con la cual asociamos las funciones

$$
\begin{gathered}
l_{\varepsilon}(\xi)=\frac{|\xi|^{2}}{2}-\phi_{\varepsilon}(|\xi|), \\
\psi_{\varepsilon}\left(\xi^{*}\right)=l_{\varepsilon}^{*}\left(\xi^{*}\right)-\frac{\left|\xi^{*}\right|^{2}}{2} .
\end{gathered}
$$

La función $\psi_{\varepsilon}$ tiene la mismas propiedades de $\psi$. En efecto, $\phi_{\varepsilon}$ es par y de clase $C^{2}$. Además, es no decreciente en $\mathbb{R}^{+}$.

Si modificamos la condición $(\widehat{H} 3$ ) reemplazándola por

( $\widehat{H} 3$ ) Existe $\varepsilon_{0}$, con $0<\varepsilon_{0}<1$, tal que, $0<\phi^{\prime \prime}(t)<1-\varepsilon_{0}$, para todo $t \in \mathbb{R}$.

Esta condición no es restrictiva, por que siempre podemos cambiar el parámetro de carga en la energía $J_{\alpha}(f)$.

Con las consideraciones anteriores enunciamos la siguiente proposición.

\section{Proposición 4.3.}

Si $\phi_{\varepsilon}$ verifica la condición (20), entonces

i) Para $0<\varepsilon<\varepsilon_{0}$, la función $\xi^{*} \rightarrow \psi_{\varepsilon}\left(\xi^{*}\right)$ es estrictamente convexa, 


$$
\text { ii) } \begin{aligned}
-b_{1}+\frac{a_{1}^{2}}{2(1-\varepsilon)}+\frac{a_{1}}{1-\varepsilon}\left|\xi^{*}\right|+\frac{\varepsilon}{2(1-\varepsilon)}\left|\xi^{*}\right|^{2} \leq \psi_{\varepsilon}\left(\xi^{*}\right) \\
\leq b_{2}+\frac{a_{2}^{2}}{2(1-\varepsilon)}+\frac{a_{2}}{1-\varepsilon}\left|\xi^{*}\right|+\frac{\varepsilon}{2(1-\varepsilon)}\left|\xi^{*}\right|^{2} .
\end{aligned}
$$

\section{Prueba.}

Ver Osorio [5], Pág. 82.

El problema regularizado asociado con $T(b)$ es

$$
\inf _{b \in L_{2}^{2}}\left\{T_{\varepsilon}(b)=\int_{\Omega}\left(\left(f_{b}-p\right)^{2}+\frac{\left|b-\nabla f_{b}\right|^{2}}{2}+\psi_{\varepsilon}(b)+\frac{\varepsilon|b|^{2}}{2}\right) d x d y\right\}
$$

para el cual establecemos la siguiente proposición.

\section{Proposición 4.4 .}

Sea $\phi_{\varepsilon}(t)=\phi(t)+\frac{\varepsilon}{2} t^{2}$, donde $\phi(t)$ satisface las condiciones (H3) y (H5) y sean las funciones asociadas

$$
l_{\varepsilon}(\xi)=\frac{|\xi|^{2}}{2}-\phi_{\varepsilon}(|\xi|), \Phi_{\varepsilon}(u)=\int_{\Omega} \phi_{\varepsilon}(|u(x, y)|) d x d y \text { y } \Psi_{\varepsilon}(u)=\int_{\Omega} \psi_{\varepsilon}(b(x, y)) d x d y
$$

Entonces,

$$
\begin{aligned}
& \Phi_{\varepsilon}(u)=\inf _{v \in L_{2}^{2}} \int_{\Omega}\left(\frac{|u-v|^{2}}{2}+\psi_{\varepsilon}(v)\right) d x d y \\
& \Psi_{\varepsilon}(v)=\operatorname{Sup}_{u \in L_{2}^{2}} \int_{\Omega}\left(-\frac{|u-v|}{2}+\phi_{\varepsilon}(|u|)\right) d x d y
\end{aligned}
$$

\section{Prueba.}

Ver Osorio [5], Pág. 84.

\section{Proposición 4.5.}

Bajo las asunciones ( $\widehat{H} 1)$, ( $\widehat{H} 2)$ y (20), el problema (21) tiene una única solución $b_{\varepsilon}$ $y$ existe una constante $c$, independiente de $\varepsilon$, tal que 


$$
\left\{\begin{array}{c}
\varepsilon\left\|b_{\varepsilon}\right\|_{L_{2}^{2}}^{2} \leq c \\
\varepsilon\left\|\nabla f_{b_{c}}\right\|_{L_{2}^{2}}^{2} \leq c \\
\left\|b_{\varepsilon}\right\|_{L_{2}^{1}} \leq c \\
\left\|f_{b_{\varepsilon}}\right\|_{L_{1}^{2}} \leq c
\end{array}\right.
$$

\section{Prueba.}

Afirmamos que el funcional $T_{\varepsilon}(b)$ es estrictamente convexo, para esto probaremos que:

a) $J_{b}(f)=\int_{\Omega}\left(|f-p|^{2}+\frac{|b-\nabla f|^{2}}{2}\right) d x d y$ es estrictamente convexo.

b) La aplicación $b \rightarrow f_{b}$ es afín de $L^{2}(\Omega)$ a $H^{1}(\Omega)$ y

c) $\Lambda(b)=\int_{\Omega} \psi_{\varepsilon}(b) d x d y$ es estrictamente convexa.

En efecto:

a) $J_{b}(f)$ es estrictamente convexa.

Pues $g(t)=t^{2}$ es estrictamente convexa por que

$$
\begin{aligned}
& {[t a+(1-t) b]^{2}<t a^{2}+(1-t) b^{2} ; 0<t<1} \\
& \Leftrightarrow t^{2} a^{2}+(1-t)^{2} b^{2}+2 t(1-t) a b<t a^{2}+(1-t) b^{2} \\
& \left(t^{2}-t\right) a^{2}+\left[(1-t)^{2}-(1-t)\right] b^{2}+2 t(1-t) a b<0 \\
& (1-t)\left[-t a^{2}-t b^{2}+2 t a b\right]<0 \\
& -t(1-t)(a-b)^{2}<0 ; \quad \forall t \in<0, \quad 1>.
\end{aligned}
$$

De aquí,

$$
\begin{aligned}
J_{b}\left(t f_{1}+(1-t) f_{2}\right) & =\int_{\Omega}\left[\left|t f_{1}+(1-t) f_{2}-p\right|^{2}+\frac{\left|b-\nabla\left(t f_{1}+(1-t) f_{2}\right)\right|^{2}}{2}\right] \\
& =\int_{\Omega}\left|t\left(f_{1}-p\right)+(1-t)\right|^{2}+\frac{\left|t\left(b-\nabla f_{1}\right)+(1-t)\left(b-\nabla f_{2}\right)\right|^{2}}{2} \\
& \leq \int_{\Omega} t\left|f_{1}-p\right|^{2}+(1-t)\left|f_{2}-p\right|^{2}+\frac{t\left|b-\nabla f_{1}\right|^{2}}{2}+(1-t) \frac{\left|b-\nabla f_{2}\right|^{2}}{2} \\
& =t J_{b}\left(f_{1}\right)+(1-t) J_{b}\left(f_{2}\right) .
\end{aligned}
$$


Asi,

$J_{b}\left(t f_{1}+(1-t) f_{2}\right) \leq t J_{b}\left(f_{1}\right)+(1-t) J_{b}\left(f_{2}\right)$, es estricto cuando $0<t<1$.

b) Las funciones $b_{i} \rightarrow f_{b_{i}}, i=1,2$ satisfacen la ecuación de Euler, esto es

$$
-\Delta f_{b_{i}}+2 f_{b_{i}}=2 p-\operatorname{div} b_{i}
$$

Entonces,

$$
\begin{aligned}
& -\Delta t f_{b_{1}}+2 t f_{b_{1}}=2 t p-\operatorname{div}\left(t b_{1}\right) \\
& -\Delta(1-t) f_{b_{2}}+2(1-t) f_{b_{2}}=2(1-t) p-\operatorname{div}\left((1-t) b_{2}\right) .
\end{aligned}
$$

Sumando (25) y (26)

$$
-\Delta\left[t f_{b_{1}}+(1-t) f_{b_{2}}\right]+2\left[t f_{b_{1}}+(1-t) f_{b_{2}}\right]=2 p-\operatorname{div}\left[t b_{1}+(1-t) b_{2}\right] .
$$

Luego,

$$
\gamma=t b_{1}+(1-t) b_{2} \rightarrow t f_{b_{1}}+(1-t) f_{b_{2}}
$$

como,

$$
\inf _{f} J_{b}(f)=J_{b}\left(f_{b}\right) \Leftrightarrow-\Delta f_{b}+2 f_{b}=2 p-\operatorname{div}(b)
$$

Entonces,

$$
J_{\gamma}\left(f_{\gamma}\right)=J_{\gamma}\left(t f_{b_{1}}+(1-t) f_{b_{2}}\right)
$$

donde $\gamma=t b_{1}+(1-t) b_{2}$.

Pero, la ecuación de Euler tiene una única solución, entonces

$f_{t b_{1}+(1-t) b_{2}}=t f_{b_{1}}+(1-t) f_{b_{2}}$ y como $J_{r}(f)$ es estrictamente convexa, entonces

$$
J_{\gamma}\left(t f_{b_{1}}+(1-t) f_{b_{2}}\right)<t J_{\gamma}\left(f_{b_{1}}\right)+(1-t) J_{\gamma}\left(f_{b_{2}}\right)
$$

c) $\Lambda(b)=\int_{\Omega} \psi_{\varepsilon}(b)$ es estrictamente convexo por que $\psi_{\varepsilon}$ lo es.

Finalmente, probaremos que $T_{\varepsilon}(b)$ es estrictamente convexo,

$$
T_{\varepsilon}\left(t b_{1}+(1-t) b_{2}\right)=J_{\gamma}\left(f_{\gamma}\right)+\Lambda(\gamma) \text {, donde } \gamma=t b_{1}+(1-t) b_{2}
$$




$$
\begin{aligned}
& T_{t_{\varepsilon}}\left(t b_{1}+(1-t) b_{2}\right)=\int\left|f_{\gamma}-p\right|^{2}+\frac{\left|\gamma-\nabla f_{\gamma}\right|^{2}}{2}+\Delta \text {, donde } \Delta=t \Lambda\left(b_{1}\right)+(1-t) \Lambda\left(b_{2}\right) \\
& =\int_{\Omega}\left|t f_{b_{1}}+(1-t) f_{b_{2}}-p\right|^{2}+\frac{\left|\gamma-\nabla\left(t f_{b_{1}}+(1-t) f_{b_{2}}\right)\right|^{2}}{2}+\Delta \\
& =\int_{\Omega}\left|t f_{b_{1}}+(1-t) f_{b_{2}}-p\right|^{2}+\frac{\left|t b_{1}+(1-t) b_{2}-\nabla\left(t f_{b_{1}}+(1-t) f_{b_{2}}\right)\right|^{2}}{2}+\Delta \\
& =\int_{\Omega}\left|t\left(f_{b_{1}}-p\right)+(1-t)\left(f_{b_{2}}-p\right)\right|^{2}+\frac{\left|t\left(b_{1}-\nabla f_{b_{1}}\right)+(1-t)\left(b_{2}-\nabla f_{b_{2}}\right)\right|^{2}}{2}+\Delta \\
& =\int_{\Omega}\left|t\left(f_{b_{1}}-p\right)+(1-t)\left(f_{b_{2}}-p\right)\right|^{2}+\frac{\left|t\left(b_{1}-\nabla f_{b_{1}}\right)+(1-t)\left(b_{2}-\nabla f_{b_{2}}\right)\right|^{2}}{2}+\Delta \\
& =t \int_{\Omega}\left|f_{b_{1}}-p\right|^{2}+\frac{\left|b_{1}-\nabla f_{b_{1}}\right|^{2}}{2}+(1-t) \int_{\Omega}\left|f_{b_{2}}-p\right|^{2}+\frac{\left|b_{2}-\nabla f_{b_{2}}\right|^{2}}{2}+\Delta \\
& =t J_{b_{1}}\left(f_{b_{1}}\right)+(1-t) J_{b_{2}}\left(f_{b_{2}}\right)+\Delta ; \text { donde } \Delta=t \Lambda\left(b_{1}\right)+(1-t) \Lambda\left(b_{2}\right) .
\end{aligned}
$$

Entonces,

$$
\begin{gathered}
T_{\mathcal{\varepsilon}}\left(t b_{1}+(1-t) b_{2}\right) \leq t\left[J_{b_{1}}\left(f_{b_{1}}\right)+\Lambda\left(b_{1}\right)\right]+(1-t)\left[J_{b_{2}}\left(f_{b_{2}}\right)+\Lambda\left(b_{2}\right)\right] \\
T_{\mathcal{E}}\left(t b_{1}+(1-t) b_{2}\right) \leq t T_{\varepsilon}\left(b_{1}\right)+(1-t) T_{\varepsilon}\left(b_{2}\right),
\end{gathered}
$$

estrictamente convexa si $0<t<1$.

Sabiendo que $T_{\varepsilon}(b)$ es estrictamente convexo, probaremos la afirmación de la proposición.

De la parte ii) de la proposición 4.3, para cada $b \in L^{2}(\Omega)^{2}$ existen constantes $a_{1}^{\prime}>0$ y $b_{1}^{\prime}$ tales que,

$$
\frac{\varepsilon}{2}\|b\|_{L_{2}^{2}}^{2}+a_{1}^{\prime}\|b\|_{L_{2}^{1}}-b_{1}^{\prime} \leq T_{\varepsilon}(b)
$$

Por lo tanto, para $\varepsilon$ fijo, la sucesión minimizante $b_{\varepsilon}^{n}$ de (21) está acotada en $L^{2}(\Omega)^{2}$. Por consiguiente, existe $b_{\varepsilon} \in L^{2}(\Omega)^{2}$; y una subsucesión denotada también por $b_{\varepsilon}$, tal que, $b_{\varepsilon}^{n} \stackrel{w}{\longrightarrow} b_{\varepsilon}$ en $L^{2}(\Omega)$ débilmente.

Debido a la convexidad estricta de $T_{\varepsilon}, b_{\varepsilon}$ es única, y toda la sucesión $b_{\varepsilon}^{\prime \prime}$ converge a $b_{\varepsilon}$. Además, 


$$
T_{\varepsilon}\left(b_{\varepsilon}\right) \leq \lim _{n \rightarrow \infty} T_{\varepsilon}\left(b_{\varepsilon}^{n}\right)=\inf T_{\varepsilon}(b) \leq T_{\varepsilon}(b) \quad ; \quad \forall b \in L_{2}^{2}
$$

Así, $b_{\varepsilon}$ es la única solución de (21)

$$
\begin{aligned}
& \int_{\Omega}\left(\left(f_{b_{\varepsilon}}-p\right)^{2}+\frac{\left|b_{\varepsilon}-\nabla f_{b_{\varepsilon}}\right|^{2}}{2}+\psi_{\varepsilon}\left(b_{\varepsilon}\right)+\frac{\varepsilon}{2}\left|b_{\varepsilon}\right|^{2}\right) d x d y \\
& \leq \int_{\Omega}\left(\left(f_{b}-p\right)^{2}+\frac{\left|b-\nabla f_{b}\right|^{2}}{2}+\psi_{\varepsilon}(b)+\frac{\varepsilon}{2}|b|^{2}\right) d x d y, \forall b \in L_{2}^{2} .
\end{aligned}
$$

Eligiendo, por ejemplo, $b=0$ en (24) es claro que; existe una constante $c$ independiente de $\varepsilon$, tal que (22) se verifica.

El siguiente teorema deriva la condición de optimalidad satisfecha por $b_{\varepsilon}$.

\section{Teorema 4.6.}

La solución $b_{\varepsilon}$ del problema (21) verifica la condición de optimalidad

$$
b_{\varepsilon}+\nabla \psi_{\varepsilon}\left(b_{\varepsilon}\right)-\nabla f_{b_{\varepsilon}}=0 \text { en casi todo punto de } \Omega \text {. }
$$

\section{Prueba.}

Para simplificar las notaciones, denotamos $f_{\varepsilon}=f_{b_{\varepsilon}}$; entonces consideramos una variación de $b_{\varepsilon}$ de la forma $b_{\theta}=b_{\varepsilon}+\theta q$, donde $\theta \in \mathbb{R}$ y $q \in L_{2}^{2}$. Denotando $f_{\theta}=f_{b_{\theta}}$, es claro, gracias a la linealidad de la fórmula (17), que

$$
f_{\theta}=f_{\varepsilon}+\theta h
$$

donde $h$ verifica

$$
-\Delta h+2 h=-\operatorname{div} q \text { en } H^{1}(\Omega)^{\prime}\left(\text { el dual de } H^{1}(\Omega)\right) .
$$

Con esta observación,

$$
\begin{aligned}
T_{\varepsilon}\left(b_{\theta}\right) & =\int_{\Omega}\left(\left(f_{b_{\theta}}-p\right)^{2}+\frac{\left|b_{\theta}-\nabla f_{\theta}\right|^{2}}{2}+\psi_{\varepsilon}\left(b_{\theta}\right)\right) d x d y \\
& =\int_{\Omega}\left(\left(\left(f_{\varepsilon}+\theta h\right)-p\right)^{2}+\frac{\left|\left(b_{\varepsilon}+\theta q\right)-\nabla\left(f_{\varepsilon}+\theta h\right)\right|^{2}}{2}+\psi_{\varepsilon}\left(b_{\varepsilon}+\theta\right)\right) d x d y . \\
T_{\varepsilon}\left(b_{\varepsilon}\right) & =\int_{\Omega}\left(\left(f_{\varepsilon}-p\right)^{2}+\frac{\left|b_{\varepsilon}-\nabla f_{\varepsilon}\right|^{2}}{2}+\psi_{\varepsilon}\left(b_{\varepsilon}\right)\right) d x d y .
\end{aligned}
$$


Cuando hacemos $T_{\varepsilon}\left(b_{\theta}\right)-T_{\varepsilon}\left(b_{\varepsilon}\right)$ se tiene en el integrando las siguientes diferencias

(1) $\left[\left(f_{\varepsilon}+\theta h\right)-p\right]^{2}-\left(f_{\varepsilon}-p\right)^{2}=2 \theta f_{\varepsilon} h+\theta^{2} h^{2}-2 \theta h p=\theta\left(2 f_{\varepsilon}-2 p+\theta h\right) h$

(2) $\left|\left(b_{\varepsilon}+\theta q\right)-\nabla\left(f_{\varepsilon}+\theta h\right)\right|^{2}-\left|b_{\varepsilon}-\nabla f_{\varepsilon}\right|^{2}=$

$$
\begin{aligned}
&=\left\langle b_{\varepsilon}+\theta q-\nabla\left(f_{\varepsilon}+\theta h\right), \quad b_{\varepsilon}+\theta q-\nabla\left(f_{\varepsilon}+\theta h\right)\right\rangle-\left\langle b_{\varepsilon}-\nabla f_{\varepsilon}, \quad b_{\varepsilon}-\nabla f_{\varepsilon}\right\rangle \\
&=\left\langle\left(b_{\varepsilon}-\nabla f_{\varepsilon}\right)+\theta(q-\nabla h), \quad\left(b_{\varepsilon}-\nabla f_{\varepsilon}\right)+\theta(q-\nabla h)\right\rangle-\left\langle b_{\varepsilon}-\nabla f_{\varepsilon}, \quad b_{\varepsilon}-\nabla f_{\varepsilon}\right\rangle \\
&=\left\langle b_{\varepsilon}-\nabla f_{\varepsilon}, \quad b_{\varepsilon}-\nabla f_{\varepsilon}\right\rangle+\left\langle b_{\varepsilon}-\nabla f_{\varepsilon}, \theta(q-\nabla h)\right\rangle+\left\langle\theta(q-\nabla h), \quad b_{\varepsilon}-\nabla f_{\varepsilon}\right\rangle+ \\
& \quad+\langle\theta(q-\nabla h), \quad \theta(q-\nabla h)\rangle-\left\langle b_{\varepsilon}-\nabla f_{\varepsilon}, \quad b_{\varepsilon}-\nabla f_{\varepsilon}\right\rangle \\
&= 2 \theta\left\langle b_{\varepsilon}-\nabla f_{\varepsilon}, \quad q-\nabla h\right\rangle+\theta^{2}\langle q-\nabla h, q-\nabla h\rangle \\
&=\theta\left\langle 2 b_{\varepsilon}-2 \nabla f_{\varepsilon}+\theta(q-\nabla h), \quad q-\nabla h\right\rangle .
\end{aligned}
$$

(3) $\psi_{\varepsilon}\left(b_{\varepsilon}+\theta q\right)-\psi_{\varepsilon}\left(b_{\varepsilon}\right)$.

Luego,

$$
\begin{aligned}
& \Delta=\frac{T_{\varepsilon}\left(b_{\theta}\right)-T_{\varepsilon}\left(b_{\varepsilon}\right)}{\theta}=\frac{1}{\theta}\left\{\int_{\Omega} \theta\left(2 f_{\varepsilon}-2 p+\theta h\right) h d x d y+\int_{\Omega} \theta\left\langle 2 b_{\varepsilon}-2 \nabla f_{\varepsilon}+\theta(q-\nabla h), q-\nabla h\right\rangle d x d y\right\} \\
& \quad+\frac{1}{\theta} \int_{\Omega}\left(\psi\left(b_{\varepsilon}+\theta q\right)-\psi\left(b_{\varepsilon}\right)\right) d x d y \\
& =\int_{\Omega}\left(2 f_{\varepsilon}-2 p+\theta h\right) h d x d y+\int_{\Omega}\left\langle 2 b_{\varepsilon}-2 \nabla f_{\varepsilon}+\theta(q-\nabla h), q-\nabla h\right\rangle d x d y \\
& \quad+\frac{1}{\theta} \int_{\Omega}\left(\psi\left(b_{\varepsilon}+\theta q\right)-\psi\left(b_{\varepsilon}\right)\right) d x d y .
\end{aligned}
$$

Por el teorema de Tahraoui, en la tercera integral el gradiente de $\nabla \psi_{\varepsilon}\left(b_{\varepsilon}\right)$ (Osorio [5], Pág. 42) satisface las condiciones para la convergencia dominada de Lebesgue. Tomando límite cuando $\theta \rightarrow 0$, el cociente incremental $\Delta$ converge a

$2 \int_{\Omega}\left(f_{\varepsilon}-p\right) h+\left\langle b_{\varepsilon}-\nabla f_{\varepsilon}, q-\nabla h\right\rangle d x d y+\int_{\Omega} \lim _{\theta \rightarrow 0}\left[\frac{\psi_{\varepsilon}\left(b_{\varepsilon}+\theta q\right)-\psi_{\varepsilon}\left(b_{\varepsilon}\right)}{\theta}\right] d x d y$.

Esto es,

$$
\begin{aligned}
\lim _{\theta \rightarrow 0} \Delta= & 2 \int_{\Omega}\left(f_{\varepsilon}-p\right) h+\left\langle b_{\varepsilon}-\nabla f_{\varepsilon}, q-\nabla h\right\rangle d x d y+\int_{\Omega}\left\langle\nabla \psi_{\varepsilon}\left(b_{\varepsilon}\right), q\right\rangle d x d y \\
& 2 \int_{\Omega}\left(f_{b}-p\right) f=\int_{\Omega}\left\langle\nabla f_{b}-b, \nabla f_{\varepsilon}\right\rangle d x d y .
\end{aligned}
$$

Tomando $f=h$ y $f_{b}=f_{\varepsilon}$, se tiene 


$$
\begin{aligned}
& 2 \int_{\Omega}\left(f_{\varepsilon}-p\right) h d x d y=\int_{\Omega}\left\langle\nabla f_{\varepsilon}-b_{\varepsilon}, \nabla h\right\rangle d x d y . \\
\lim _{\theta \rightarrow 0} \Delta= & -\int_{\Omega}\left\langle\nabla f_{\varepsilon}-p, \nabla h\right\rangle d x d y+2 \int_{\Omega}\left\langle b_{\varepsilon}-\nabla f_{\varepsilon}, q-\nabla h\right\rangle d x d y+\int_{\Omega}\left\langle\nabla \psi_{\varepsilon}\left(b_{\varepsilon}\right), q\right\rangle d x d y \\
= & \int_{\Omega}\left[\left\langle b_{\varepsilon}-\nabla f_{\varepsilon}, \nabla h\right\rangle+2\left\langle b_{\varepsilon}-\nabla f_{\varepsilon}, q-\nabla h\right\rangle\right] d x d y+\int_{\Omega}\left\langle\nabla \psi_{\varepsilon}\left(b_{\varepsilon}\right), q\right\rangle d x d y \\
= & \int_{\Omega}\left\langle b_{\varepsilon}-\nabla f_{\varepsilon}, \nabla h+2 q-2 \nabla h\right\rangle d x d y+\int_{\Omega}\left\langle\nabla \psi_{\varepsilon}\left(b_{\varepsilon}\right), q\right\rangle d x d y \\
= & \int_{\Omega}\left\langle b_{\varepsilon}-\nabla f_{\varepsilon},-\nabla h+2 q\right\rangle d x d y+\int_{\Omega}\left\langle\nabla \psi_{\varepsilon}\left(b_{\varepsilon}\right), q\right\rangle d x d y .
\end{aligned}
$$

Luego, como $\Delta h=\operatorname{div} q$, entonces

$$
-\nabla h=-q
$$

y de aquí,

$$
\begin{aligned}
\lim _{\theta \rightarrow 0} \Delta & =\int_{\Omega}\left\langle b_{\varepsilon}-\nabla f_{\varepsilon}, q\right\rangle d x d y+\int_{\Omega}\left\langle\nabla \psi_{\varepsilon}\left(b_{\varepsilon}\right), q\right\rangle d x d y \\
& =\int_{\Omega}\left\langle b_{\varepsilon}-\nabla f_{\varepsilon}+\nabla \psi_{\varepsilon}\left(b_{\varepsilon}\right), q\right\rangle d x d y .
\end{aligned}
$$

Pero como

$$
\Delta=\frac{T_{\varepsilon}\left(b_{\theta}\right)-T_{\varepsilon}\left(b_{\varepsilon}\right)}{\theta}=\frac{T_{\varepsilon}\left(b_{\varepsilon}+\theta q\right)-T_{\varepsilon}\left(b_{\varepsilon}\right)}{\theta}
$$

$\lim _{\theta \rightarrow 0} \Delta=0$ por que $b_{\varepsilon}$ es punto crítico de $T_{\varepsilon}$

$$
\Rightarrow \int_{\Omega}\left\langle b_{\varepsilon}-\nabla f_{\varepsilon}+\nabla \psi_{\varepsilon}\left(b_{\varepsilon}\right), q\right\rangle d x d y=0 ; \forall q \in\left(L^{2}(\Omega)\right)^{2}
$$

De aquí obtenemos,

$$
b_{\varepsilon}-\nabla f_{\varepsilon}+\nabla \psi_{\varepsilon} \quad\left(b_{\varepsilon}\right)=0 \text { en casi todo punto de } \Omega
$$

que es la relación que deseábamos.

En el siguiente corolario, expresamos $\nabla \psi_{\varepsilon}\left(b_{\varepsilon}\right)$ en términos de $\nabla f_{\varepsilon}$ y $\phi^{\prime}\left(\left|\nabla f_{\varepsilon}\right|\right)$.

\section{Corolario 4.7.}

La condición de optimalidad (31) puede ser escrito como 


$$
b_{\varepsilon}=\left((1-\varepsilon)-\frac{\phi^{\prime}\left(\left|\nabla f_{\varepsilon}\right|\right)}{\left|\nabla f_{\varepsilon}\right|}\right) \nabla f_{\varepsilon} .
$$

\section{Prueba.}

Sabemos que

$$
l_{\varepsilon}(\xi)=\frac{|\xi|^{2}}{2}-\phi(|\xi|)-\frac{\varepsilon}{2}|\xi|^{2}
$$

Por ( $\widehat{H} 2$ ) y (20), la función $l_{\varepsilon}$ es estrictamente convexa; por lo tanto, $l_{\varepsilon}^{*}$ es diferenciable.

Además, $l_{\varepsilon}^{*}\left(\xi^{*}\right)=\operatorname{Sup}_{\xi}\left(\left\langle\xi^{*}, \xi\right\rangle-l_{\varepsilon}(\xi)\right)$, luego $l_{\varepsilon}^{*}\left(\xi^{*}\right)=\left\langle\xi^{*}, \xi_{\varepsilon}\right\rangle-l_{\varepsilon}\left(\xi_{\varepsilon}\right)$, donde, $\xi_{\varepsilon}$ es el único punto donde la función $\left\langle\xi^{*}, \xi\right\rangle-l_{\varepsilon}(\xi)$ alcanza su máximo.

De aquí,

$$
\nabla l_{\varepsilon}^{*}\left(\xi^{*}\right)=\xi_{\varepsilon} \quad\left(\text { derivando respecto de } \xi^{*}\right)
$$

De otro lado, $\left\langle\xi^{*}, \xi\right\rangle-l_{\varepsilon}(\xi)$ como función de la variable $\xi$ alcanza su máximo en $\xi_{\varepsilon}$. Luego,

$$
\xi^{*}-\nabla l_{\varepsilon}\left(\xi_{\varepsilon}\right)=0 \text { (derivando respecto de } \xi \text { ). }
$$

De aquí, tomando en cuenta la definición de $l_{\varepsilon}(\xi)$

$$
\begin{aligned}
& \xi^{*}-\nabla\left(\frac{(1-\varepsilon)}{2}\left|\xi_{\varepsilon}\right|^{2}-\phi\left(\left|\xi_{\varepsilon}\right|\right)\right)=0 \\
& \xi^{*}-(1-\varepsilon) \xi_{\varepsilon}+\varphi^{\prime}\left(\left|\xi_{\varepsilon}\right|\right) \frac{\xi_{\varepsilon}}{\left|\xi_{\varepsilon}\right|}=0 \\
& \xi^{*}=(1-\varepsilon) \xi_{\varepsilon}-\varphi^{\prime}\left(\left|\xi_{\varepsilon}\right|\right) \frac{\xi_{\varepsilon}}{\left|\xi_{\varepsilon}\right|} .
\end{aligned}
$$

De otro lado, considerando que $l_{\varepsilon}$ es estrictamente convexo, entonces la función

$$
\psi_{\varepsilon}\left(\xi^{*}\right)=l_{\varepsilon}^{*}\left(\xi^{*}\right)-\frac{\left|\xi^{*}\right|^{2}}{2}
$$

es diferenciable.

Luego,

$$
\nabla \psi_{\varepsilon}\left(\xi^{*}\right)=\nabla l_{\varepsilon}^{*}\left(\xi^{*}\right)-\xi^{*} \text { o sea }
$$




$$
\nabla l_{\varepsilon}^{*}\left(\xi^{*}\right)=\nabla \psi_{\varepsilon}\left(\xi^{*}\right)+\xi^{*}
$$

Denotando

$$
L_{\varepsilon}(\xi)=(1-\varepsilon) \xi-\xi \frac{\phi^{\prime}\left(\left|\xi_{\varepsilon}\right|\right)}{\left|\xi_{\varepsilon}\right|}
$$

gracias a (20), $L_{\varepsilon}$ es invertible y (34) nos dice que $\xi^{*}=L\left(\xi_{\varepsilon}\right)$, de aqui

$$
\xi_{\varepsilon}=L^{-1}\left(\xi^{*}\right) \text { y como } \xi_{\varepsilon}=\nabla l_{\varepsilon}^{*}\left(\xi^{*}\right) \text {, tomando en cuenta (35) nos da }
$$

$$
\nabla \psi_{\varepsilon}\left(\xi^{*}\right)=L^{-1}\left(\xi^{*}\right)
$$

Consiảerando la condición de optimalidad, y de (28) tenemos la siguiente sucesión de igualdades:

$$
\begin{aligned}
\nabla \psi_{\varepsilon}\left(b_{\varepsilon}\right)+b_{\varepsilon}-\nabla f_{\varepsilon} & =0, \\
L^{-1}\left(b_{\varepsilon}\right)-b_{\varepsilon}+b_{\varepsilon}-\nabla f_{\varepsilon} & =0, \\
L^{-1}\left(b_{\varepsilon}\right) & =\nabla f_{\varepsilon},
\end{aligned},
$$

de aqui

$$
\begin{aligned}
& b_{\varepsilon}=L\left(\nabla f_{\varepsilon}\right)=(1-\varepsilon) \nabla f_{\varepsilon}-\nabla f_{\varepsilon} \frac{\phi^{\prime}\left(\left|\nabla f_{\varepsilon}\right|\right)}{\left|\nabla f_{\varepsilon}\right|} \\
& b_{\varepsilon}=\left((1-\varepsilon)-\frac{\phi^{\prime}\left(\left|\nabla f_{\varepsilon}\right|\right)}{\left|\nabla f_{\varepsilon}\right|}\right) \nabla f_{\varepsilon} .
\end{aligned}
$$

Ahora, probaremos la existencia de una solución para el problema inicial

$$
\begin{aligned}
\nabla \psi\left(\xi^{*}\right) & =\xi_{0}-\nabla l\left(\xi_{0}\right)=-\nabla(\phi|\xi|)_{\xi=\xi_{0}} \\
& =-\frac{\phi^{\prime}\left(\left|\xi_{0}\right|\right)}{\left|\xi_{0}\right|} \xi_{0} .
\end{aligned}
$$

Queda por estudiar el comportamiento de $f_{\varepsilon}$ y $b_{\varepsilon}$ cuando $\varepsilon \rightarrow 0$. El sistema que liga $f_{\varepsilon}$ y $b_{\varepsilon}$ consiste de las ecuaciones, (17) y (31).

De (31) tenemos

$$
\operatorname{div} b_{\varepsilon}=\left((1-\varepsilon) \Delta f_{\varepsilon}-\operatorname{div}\left(\frac{\phi^{\prime}\left(\left|\nabla f_{\varepsilon}\right|\right)}{\left|\nabla f_{\varepsilon}\right|}\right) \nabla f_{\varepsilon}\right) \text {. }
$$

Poniendo esta ecuación en (17), obtenemos 


$$
\varepsilon \Delta f_{\varepsilon}+\operatorname{div}\left(\frac{\phi^{\prime}\left(\left|\nabla f_{\varepsilon}\right|\right)}{\left|\nabla f_{\varepsilon}\right|}\right) \nabla f_{\varepsilon}=2\left(f_{\varepsilon}-p\right)
$$

Entonces la solución de (31) es exactamente el minimizante del funcional, $J_{\varepsilon}(f)$, es decir,

$$
J_{\varepsilon}\left(f_{\varepsilon}\right)=\inf \left\{J_{\varepsilon}(f) ; f \in H^{1}(\Omega)\right\},
$$

donde

$$
J_{\varepsilon}(f)=\int_{\Omega}(p-f)^{2} d x d y+\int_{\Omega} \phi(|\nabla f|) d x d y+\frac{\varepsilon}{2} \int_{\Omega}|\nabla f|^{2} d x d y
$$

Por otra parte, observamos, gracias a (29) y (21), que

$$
\begin{aligned}
J_{\varepsilon}(f) & =\int_{\Omega}\left(p-f_{\varepsilon}\right)^{2} d x d y+\int_{\Omega} \phi\left(\left|\nabla f_{\varepsilon}\right|\right) d x d y+\frac{\varepsilon}{2} \int_{\Omega}\left|\nabla f_{\varepsilon}\right|^{2} d x d y \\
& =\int_{\Omega}\left(p-f_{\varepsilon}\right)^{2} d x d y+\inf _{b \in L^{2}(\Omega)^{2}} \int_{\Omega}\left(\frac{\left|b-D f_{\varepsilon}\right|^{2}}{2}+\psi_{\varepsilon}(b)\right) d x d y \\
& =\int_{\Omega}\left(\left(p-f_{\varepsilon}\right)^{2}+\left(\frac{\left|b_{\varepsilon}-\nabla f_{\varepsilon}\right|^{2}}{2}+\psi_{\varepsilon}\left(b_{\varepsilon}\right)\right) d x d y\right. \\
& =\inf _{b \in L^{2}(\Omega)^{2}} T_{\varepsilon}(b) \leq J_{\varepsilon}(f) ; \forall f \in H^{1}(\Omega) .
\end{aligned}
$$

Por los resultados clásicos de regularidad, la solución $f_{\varepsilon}$ de (31) pertenece a $C^{2}(\Omega)$. (Ver Gilbarg and Trudinger [4]).

\section{Proposición 4.8.}

Si $p$ verifica ( $\widehat{H} 1$ ), entonces la solución $f_{\varepsilon}$ de (36) satisface

$$
0 \leq f_{\varepsilon}(x, y) \leq 1 \quad \text { a.e. }(x, y) \in \Omega
$$

\section{Prueba.}

Demostraremos, que $f_{\varepsilon}(x, y) \leq 1$, a.e. $(x, y) \in \Omega$. La otra desigualdad puede ser demostrada del mismo modo.

$f_{\varepsilon}$ es una solución del problema variacional

$2 \int_{\Omega}\left(f_{\varepsilon}-p\right) v d x d y+\int_{\Omega} \frac{\phi^{\prime}\left(\left|\nabla f_{\varepsilon}\right|\right)}{\left|\nabla f_{\varepsilon}\right|}\left\langle\nabla f_{\varepsilon}, \nabla v\right\rangle d x d y+\varepsilon \int_{\Omega}\left\langle\nabla f_{\varepsilon}, \nabla v\right\rangle d x d y=0, \forall v \in H^{1}(\Omega)$ 
En (39) clegimos la parte positiva de $\left(f_{\varepsilon}-1\right)$ que denotamos por $v=\left(f_{\varepsilon}-1\right)^{+} ; v \in H^{1}(\Omega)$, 3่t nodo que, (39) puede ser escrito como

$$
\begin{aligned}
\Delta & =2 \int_{\Omega}\left(f_{\varepsilon}-p\right)\left(f_{\varepsilon}-1\right)^{+} d x d y+\int_{\Omega} \frac{\phi^{\prime}\left(\left|\nabla f_{\varepsilon}\right|\right)}{\left|\nabla f_{\varepsilon}\right|}\left\langle\nabla f_{\varepsilon}, \nabla\left(f_{\varepsilon}-1\right)^{+}\right\rangle d x d y \\
& +\varepsilon \int_{\Omega}\left\langle\nabla f_{\varepsilon}, \nabla\left(f_{\varepsilon}-1\right)^{+}\right\rangle d x d y=0,
\end{aligned}
$$

como $\left(f_{\varepsilon}-1\right)^{-}=0$ en los $\xi$, tal que, $f_{\varepsilon}(\xi)>1, \int_{\left(f_{\varepsilon}>1\right)}\left(f_{\varepsilon}-1\right)^{-}=0$, así

$$
\begin{aligned}
\Delta & =2 \int_{\left(f_{\varepsilon}>1\right)}\left(f_{\varepsilon}-p\right)\left(f_{\varepsilon}-1\right) d x d y+\int_{\left(f_{\varepsilon}>1\right)} \frac{\phi^{\prime}\left(\left|\nabla f_{\varepsilon}\right|\right)}{\left|\nabla f_{\varepsilon}\right|} \nabla f_{\varepsilon} \cdot \nabla\left(f_{\varepsilon}-1\right) d x d y \\
& +\varepsilon \int_{\left(f_{\varepsilon}>1\right)} \nabla\left(f_{\varepsilon}-1\right) \cdot \nabla\left(f_{\varepsilon}-1\right) d x d y=0 \\
& \int_{\left(f_{\varepsilon}>1\right)}\left(f_{\varepsilon}-p\right)\left(f_{\varepsilon}-1\right) d x d y+\int_{\left(f_{\varepsilon}>1\right)} \frac{\phi^{\prime}\left(\left|\nabla f_{\varepsilon}\right|\right)}{\nabla\left(f_{\varepsilon}-1\right) \mid} \nabla f_{\dot{\varepsilon}} \cdot \nabla\left(f_{\varepsilon}-1\right) d x d y \\
& +\varepsilon \int_{\left(f_{\varepsilon}>1\right)} \nabla\left(f_{\varepsilon}-1\right) \cdot \nabla\left(f_{\varepsilon}-1\right) d x d y=0
\end{aligned}
$$

Luego,

$$
\begin{aligned}
& \int_{\left(f_{\varepsilon}>1\right)} \phi^{\prime}\left(\left|\nabla\left(f_{\varepsilon}-1\right)\right|\right)\left|\nabla\left(f_{\varepsilon}-1\right)\right| d x d y+\varepsilon \int_{\left(f_{\varepsilon}>1\right)}\left|\nabla\left(f_{\varepsilon}-1\right)\right|^{2} d x d y= \\
& -2 \int_{\left(f_{\varepsilon}>1\right)}\left(f_{\varepsilon}-p\right)\left(f_{\varepsilon}-1\right) d x d y .
\end{aligned}
$$

Como $f_{\varepsilon}>1>p \Rightarrow f_{\varepsilon}-1 \geq 0$ y $f_{\varepsilon}-1>0$ en $\left(f_{\varepsilon}>1\right)$. La expresión (41) puede ser escrita como

$$
=-2 \int_{f_{\varepsilon}>1}\left(f_{\varepsilon}-p\right)\left(f_{\varepsilon}-1\right) \leq 0
$$

Luego, la relación obtenida en (41) es menor o igual que cero y es la suma de términos no negativos, entonces cada término es igual a cero, en particular

$$
\int_{\left(f_{\varepsilon}>1\right)}\left|\nabla\left(f_{\varepsilon}-1\right)\right|^{2} d x d y=0 \Rightarrow \nabla\left(f_{\varepsilon}-1\right)^{+}=0 \text {, en casi todo punto. }
$$

De aquí, $\left(f_{\varepsilon}-1\right)^{+}=c$. Pero existe $\xi$, tal que $\left(f_{\varepsilon}-1\right)^{+}(\xi)=0 \Rightarrow c=0$.

Luego, $\left(f_{\varepsilon}-1\right)^{+}=0$, y como $\left(f_{\varepsilon}-1\right)=\left(f_{\varepsilon}-1\right)^{+}-\left(f_{\varepsilon}-1\right)^{-}$

$\Rightarrow\left(f_{\varepsilon}-1\right)=-\left(f_{\varepsilon}-1\right)^{-} \leq 0 \Rightarrow f_{\varepsilon} \leq 1$. 
Con as consideraciones anteriores la relación (39) puede ser escrita como

$$
\varepsilon \int_{f_{\varepsilon}>1}\left|\nabla f_{\varepsilon}\right|^{2} d x d y+\int_{f_{\varepsilon}>1} \phi^{\prime}\left(\left|\nabla f_{\varepsilon}\right|\right) d x d y=-2 \int_{f_{\varepsilon}>1}\left(f_{\varepsilon}-p\right)\left(f_{\varepsilon}-1\right)
$$

Perc, por hipótesis, $\phi^{\prime}(t) \geq 0$ sobre $R^{+}(\operatorname{ver}(\widehat{H} 2))$ y $0 \leq p(x, y) \leq 1$ en casi todo punto de $\Omega$, entonces, $\left(f_{\varepsilon}-p\right)(x, y) \geq 0$ a.e. $(x, y) \in\left\{(x, y) ; f_{\varepsilon}>1\right\}$, el cual implica, de (42), que

$$
\int_{f_{\varepsilon}>1}\left|\nabla f_{\varepsilon}\right|^{2} d x d y \leq 0
$$

De aquí tenemos que,

$$
\nabla f_{\varepsilon}(x, y)=0, \forall(x, y) \in\left\{(x, y) ; f_{\varepsilon}>1\right\} ; \text { i.e.; } \quad\left(f_{\varepsilon}-1\right)^{+},=0 \text {; }
$$

el cual es equivalente a $f_{\varepsilon}(x, y) \leq 1$ a.e. $(x, y) \in \Omega$.

Las estimaciones siguientes son más delicadas y están basadas sobre una perturbación muy fina debido al lema de Temam (Ekeland y Teman [2], y Teman [6]), que asumiremos sin demostración.

Teorema 4.9.

Si $p \in W^{1, \infty}$, entonces para todo conjunto abierto $O$ relativamente compacto en $\Omega$, existe una constante $K=K\left(O, \Omega,\|p\|_{W^{1, \infty}}\right)$, tal que,

$$
\begin{aligned}
& \left\|f_{\varepsilon}\right\|_{W^{1, \infty}(O)} \leq K \\
& \left\|f_{\varepsilon}\right\|_{H^{2}(O)} \leq K
\end{aligned}
$$

Esta proposición nos permite el paso del límite sobre $f_{\varepsilon}$ y $b_{\varepsilon}$ cuando $\varepsilon \rightarrow 0$.Además, de las estimaciones (38), (43) y (44), podemos agregar, gracias a ( $\widehat{H} 2)$ :

$$
\left\|f_{\varepsilon}\right\|_{W^{1,1}(\Omega)} \leq C(C \text { independiente de } \varepsilon)
$$

Con estas estimaciones, usando resultados clásicos de compacidad y el de diagonalización, podemos establecer, que existe una función $f_{0}$ y una sucesión $\varepsilon_{m} \rightarrow 0$ tal que

$$
\begin{aligned}
& f_{\varepsilon_{m}} \stackrel{w^{*}}{\longrightarrow} f_{0} \text { en } L^{\infty}(\Omega) \text { débil - estrella } \\
& \nabla f_{\varepsilon_{m}} \stackrel{w^{*}}{\longrightarrow} \nabla f_{0} \text { en } L^{\infty}(O) \text { débil - estrella } \forall O \subset \bar{O} \subset \Omega \\
& f_{\varepsilon_{m}} \stackrel{w}{\longrightarrow} f_{0} \text { en } H^{2}(O) \text { débil } \forall O \subset \bar{O} \subset \Omega
\end{aligned}
$$




$$
\begin{aligned}
& f_{\varepsilon_{m}} \rightarrow f_{0} \text { en } L^{1}(\Omega) \text { fuerte, } \\
& f_{\varepsilon_{m}}\left|O \rightarrow f_{0}\right|_{O} \text { en } H^{1}(O) \text { fuerte } \forall O \subset \bar{O} \subset \Omega \\
& f_{\varepsilon_{m}}(x, y) \rightarrow f_{0}(x, y) \text { a.e. }(x, y), \\
& \nabla f_{\varepsilon_{m}}(x, y) \rightarrow \nabla f_{0}(x, y) \text { a.e. }(x, y)
\end{aligned}
$$

y tenemos el resultado siguiente.

\section{Teorema 4.10.}

Bajo las suposiciones $(\widehat{H} 1)$, ( $\widehat{H} 2)$ y $(\widehat{H} 3)$ y si $p \in W^{1, \infty}(\Omega)$, entonces la función $f_{0}$ definida anteriormente pertenece a $W^{1,1}(\Omega) \cap L^{\infty}(\Omega)$ y es la única solución del problema inicial de optimización

$$
\inf \left\{J(f)=\int_{\Omega}(p-f)^{2} d x d y+\int_{\Omega} \phi(|\nabla f|) d x d y ; f \in L^{2}(\Omega), \nabla f \in L_{2}^{1}\right\}
$$

\section{Prueba.}

Por el lema de Fatou, (45) y (52), se cumple que $f_{0}$ pertenece $W^{1,1}(\Omega) \cap L^{\infty}(\Omega)$, tenemos, además que $\left.f_{0}\right|_{O} \in H^{2}(O) \cap W^{1, \infty}(O)$, para todo $O$ con $O \subset \bar{O} \subset \Omega$. $f_{0}$ es una solución de (53). En efecto, $f_{\varepsilon_{m}}$ es la solución del problema variacional (39). Gracias a un resultado de Tahraoui mencionado antes, las condiciones $(\widehat{H} 2)$ y $(\widehat{H} 3)$ implican que existe una constante $M>0$ tal que $\left|\phi^{\prime}(t)\right| \leq M$, para todo $t \in R$. Por lo tanto, por las convergencias (46) - (52) y el teorema de la convergencia dominada de Lebesgue, podemos pasar al límite en (39) y obtener

$$
2 \int_{\Omega}\left(f_{0}-p\right) v d x d y+\int_{\Omega} \frac{\phi^{\prime}\left(\left|\nabla f_{0}\right|\right)}{\left|\nabla f_{0}\right|}\left\langle\nabla f_{0}, \nabla v\right\rangle d x d y=0, \forall v \in H^{1}(\Omega)
$$

Por argumentos de densidad, (54) es verdadero para todo $v \in L^{2}$ con $\nabla v \in L_{2}^{1}, \mathrm{y}$ desde que el problema es estrictamente convexo, $f_{0}$ es la única solución de (53). Además, $0 \leq f_{0}(x, y) \leq 1$ a.e. $(x, y) \in \Omega$.

Los resultados previos implican algunas propiedades de convergencia para la sucesión de las variables duales $b_{\varepsilon}$. En efecto, hemos probado que $b_{\varepsilon}$ verifica

$$
\begin{aligned}
& b_{\varepsilon}=\left((1-\varepsilon)-\frac{\phi^{\prime \prime}\left(\left|\nabla f_{\varepsilon}\right|\right)}{\left|\nabla f_{\varepsilon}\right|}\right) \nabla f_{\varepsilon} \quad \mathrm{y} \\
& J_{\varepsilon}\left(f_{\varepsilon}\right)=J\left(f_{\varepsilon}\right)+\frac{\varepsilon}{2} \int_{\Omega}\left|\nabla f_{\varepsilon}\right|^{2} d x d y
\end{aligned}
$$




$$
\begin{aligned}
& =\int_{\Omega}\left(p-f_{\varepsilon}\right)^{2} d x d y+\inf _{b \in L_{2}^{2}} \int_{\Omega}\left(\frac{\left|b-\nabla f_{\varepsilon}\right|^{2}}{2}+\psi_{\varepsilon}(b)\right) d x d y \\
& =\inf _{b \in L_{2}^{2}} T_{\varepsilon}(b) \leq J_{\varepsilon}(f), \quad \forall f \in H^{1}(\Omega) .
\end{aligned}
$$

Si $\varepsilon \rightarrow 0$, deducimos de (55) que

$$
b_{\varepsilon}(x, y) \rightarrow b_{0}(x, y) \text { a.e. }(x, y) \in \Omega,
$$

donde

$$
b_{0}(x, y)=\left(1-\frac{\phi^{\prime}\left(\left|\nabla f_{0}(x, y)\right|\right)}{\left|\nabla f_{0}(x, y)\right|}\right) \nabla f_{0}(x, y)
$$

La sucesión de ecuaciones en (56) prueba que $f_{\varepsilon}$ es una sucesión minimizante para el problema $\inf _{f} J(f)$, y que

$$
\begin{aligned}
\int_{\Omega} \phi\left(\left|\nabla f_{0}\right|\right) d x d y & =\lim _{\varepsilon \rightarrow 0} \inf _{b \in L_{2}^{2}} \int_{\Omega}\left(\frac{\left|b-\nabla f_{0}\right|^{2}}{2}+\psi_{\varepsilon}(b)\right) d x d y \\
& =\lim _{\varepsilon \rightarrow 0} \int_{\Omega}\left(\phi\left(\left|\nabla f_{\varepsilon}\right|\right)+\frac{\varepsilon}{2}\left|\nabla f_{\varepsilon}\right|^{2}\right) d x d y .
\end{aligned}
$$

\section{REFERENCIAS BIBLIOGRÁFICAS}

[1] G. Aubert - L. Vese, "A Variational Method in Image Recovery», SIAM J. Numer. Anal., Vol. 34, Nº 5, pp. 1948-1979, October 1997.

[2] I. Ekeland y R. Teman, "Convex Analysis and Variational Problems», NorthHolland Publishing Company, Amsterdan 1976.

[3] Pedro C. Espinoza Haro "Compresión de Imágenes en Computación Gráfica» Actas del I Seminario Internacional de Matemáticas Aplicadas, Universidad de Lima, 1998.

[4] D. Gilbarg y N.S. Trudinger, «Elliptic Partial Differential Equations of Second Order», Springer-Verlang, Berlin, 1983.

[5] Víctor G. Osorio Vidal «Convexidad y Métodos Variacionales en la Recuperación de Imágenes», Tésis para optar Grado de Magíster en Matemáticas. Pontificia Universidad Católica del Perú., 2000.

[6] R. Teman "Solutions Généralisées de Certaines Équations du Type Hypersurfaces Minima», Arch. Rational Mech. Anal., 44(1971), pp. 121-156. 